\title{
O VIRTUAL INTERNATIONAL AUTHORITY FILE - VIAF E A AGREGAÇÃO DE VALORES POR METADADOS DE AUTORIDADE
}

\author{
THE VIRTUAL INTERNATIONAL AUTHORITY FILE - VIAF AND AGGREGATION OF \\ VALUES BY AUTHORITY METADATA
}

El VIRTUAL INTERNATIONAL AUTHORITY FILE - VIAF Y LA AGREGACIÓN DE VALORES AUTORIDAD PARA METADATOS

${ }^{1}$ Luiza de Menezes Romanetto, ${ }^{1}$ Plácida Leopoldina Ventura A. da Costa Santos, ${ }^{1}$ Rachel Cristina Vesú Alves

${ }^{1}$ Universidade Estadual Paulista

\section{Correspondência}

${ }^{1}$ Luiza de Menezes Romanetto Universidade Estadual Paulista São Paulo, SP

Email: luiza.menezes@marilia.unesp.br ORCID:

Submetido em: 13-12-2016

Aceito em: 04-04-2017

Publicado: 07-06-2017

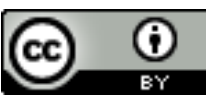

JITA: IE. Data and metadata structures. 
RESUMO: O Virtual International Authority File (VIAF) é um consórcio de cooperação internacional entre agências e bibliotecas nacionais em diversas localidades do mundo, por meio do qual tem sido estabelecida a agregação de vocabulários de valor e disponibilização de arquivos de autoridade publicados em Linked Open Data. O consórcio foi idealizado a partir de fundamentos, conceitos e tecnologias estabelecidos na catalogação que, diante de limitações tecnológicas, têm sido convertidos a partir das recomendações do World Wide Web Consortium para a publicação de dados vinculados, o que proporciona infraestrutura para o intercâmbio, o compartilhamento e a vinculação de dados de autoridade na Web de dados, além da construção de vocabulários de valor de alto nível. Este estudo tem por objetivo apresentar os fundamentos, os conceitos e as tecnologias envolvidas no desenvolvimento do VIAF. O estudo, realizado por meio de pesquisa bibliográfica e documental, apresenta como resultados a relação entre os conceitos de controle de autoridade, controle de bibliográfico, Linked Data, Linked Open Data, entre outros, com a infraestrutura estabelecida no VIAF, assim como a contribuição do consórcio na unificação de variações nacionais nas descrições de valor, por meio da formação de clusters que proporcionam controle terminológico de valores que contemplam diversidade linguística e cultural. Conclui-se que o VIAF representa uma iniciativa democrática de cooperação em nível internacional e que pode ser utilizado tanto como uma fonte confiável de arquivos de autoridade para instituições bibliotecárias, quanto para as comunidades da Web de dados.

PALABRAS-CHAVE: Virtual International Authority File - VIAF. Controle de autoridade. Metadado. Vocabulários de valor. Catalogação.

ABSTRACT: The Virtual International Authority File (VIAF) is an international cooperation consortium, which has established cooperation between national bibliographic agencies and libraries in several parts of the world and has added value and availability of authority files in Linked Open Data. The consortium was designed based on bases, concepts and technologies established in cataloging, which due to technological limitations, have been converted through World Wide Web Consortium recommendations to publish linked data. It provides infrastructure for the exchange and sharing of authority data in Web of data, in addition to the construction of value vocabularies of high level. This study aims to present and describe the bases, concepts and technologies involved in the development of VIAF. The study was accomplished through of literature documentary and shows, as a result, the relationship between concepts of authority control, bibliographic control, linked data, among others, with the established infrastructure in VIAF. Furthermore, it shows the contributions of the consortium to the unification of the national variations in descriptions of value, through cluster formation, which provides terminological control in the values that include linguistic and cultural diversity. In conclusion, the VIAF is a democratic initiative of international cooperation and can be used as a reliable source of authority files for librarian institutions, as well as to the Linked Data community.

KEYWORDS: Virtual International Authority File - VIAF. Control authority. Metadata. Vocabularies value. Cataloging.

RESUMEN: El Virtual International Authority File (VIAF) es un consorcio de cooperación internacional, entre agencias y bibliotecas nacionales en diversas localidades del mundo, a través del cual ha sido establecida la agregación de vocabularios de valor y la disponibilidad de archivos de autoridad publicados en Linked Open Data. El consorcio fue idealizado desde fundamentos, conceptos y tecnologías establecidos en la catalogación que, delante de limitaciones tecnológicas, han sido convertidos a través de recomendaciones del World Wide Web Consortium para la publicación de datos vinculados, lo que proporciona infraestructura para el intercambio, y la vinculación de datos de autoridad en la Web de datos, además de la construcción de vocabularios de valor de alto nivel. Este estudio tiene como objetivo presentar los fundamentos, los conceptos y las tecnologías involucradas en el desarrollo de VIAF. El estudio, realizado por medio de investigación bibliográfica y documental, presenta como resultados la relación entre los conceptos de control de autoridad, control bibliográfico, Linked Data, Linked Open Data (datos vinculados), entre otros, con la infraestructura establecida en VIAF, así como la contribución del consorcio en la unificación de las variaciones nacionales en las descripciones de valor, por medio de la formación de clúster que proporciona control terminológico de valores que incluyen la diversidad lingüística y cultural. En conclusión, VIAF representa una iniciativa democrática de cooperación a nivel internacional y que se puede utilizar tanto como una fuente confiable de archivos de autoridad para las instituciones bibliotecarias, como para las comunidades de la Web de datos.

PAlabras Clave: Virtual International Authority File - VIAF. Control de autoridades. Metadato. Vocabularios de valor. Catalogación.

\begin{tabular}{l|l|l} 
v.15 & $\mathrm{n} .3$ \\
\hline
\end{tabular}

p.571-590

set./dez. 2017 


\section{INTRODUÇÃ̃o}

O Virtual International Authority File (VIAF) é um consórcio internacional de cooperação estabelecido entre bibliotecas e agências nacionais que contribuem de forma mútua para a disponibilização de arquivos de autoridade em âmbito internacional. Lançado como um projeto em 1998, o consórcio foi formalizado em 2003 entre as instituições Library of Congress (LC), a Deutsche Nationalbibliothek (DNB) e o Online Computer Library Center (OCLC) (OCLC, 2015). A partir do ano de 2012, o VIAF passou a ser oferecido pela OCLC.

Os arquivos de dados de autoridade são disponibilizados no projeto Linked Open Data, sendo que o conjunto de dados publicados pelo VIAF constitui-se como conjunto de dados de autoridade de maior valor na nuvem de dados (PASTOR SANCHEZ; MARTINEZ MENDEZ; RODRIGUES MUNOZ, 2012). No projeto Linked Open Data, fundado em 2007 a comunidade internacional tem reunido esforços na publicação de dados abertos vinculados na Web de dados, pois o efetivo funcionamento do conceito estabelecido para a Web Semântica requer o acúmulo de uma vasta quantidade de dados que estejam estruturados em um formato padrão e que sejam relacionáveis (WORLD WIDE WEB CONSORTIUM, 2014).

A infraestrutura estabelecida no VIAF para a cooperação e compartilhamento de dados está de acordo com o projeto Controle Bibliográfico Universal (CBU), idealizado pela International Federation of Library Associations and Institutions - (IFLA) e adotado pela United Nations Educational, Scientific and Cultural Organization (UNESCO) durante a década de 1970. O projeto CBU desenvolvido entre as décadas de 1970 a 1990, tinha por objetivo promover o compartilhamento e o intercâmbio de registros bibliográficos e de autoridade, a fim de organizar a produção de dados bibliográficos e de controle de qualidade em uma escala universal, por meio de uma responsabilidade compartilhada que traz benefícios em relação ao retrabalho e elaboração de registros (ANGJELI; MAC EWAN; BOULET, 2014).

O projeto Controle Bibliográfico Universal segue dois princípios: 1) cada país é responsável pelo controle bibliográfico de suas publicações; 2) cada país é responsável por elaborar e disponibilizar aos demais países do mundo os registros bibliográficos e de autoridade dessas publicações (TILLET, 2000).

Os avanços tecnológicos desenvolvidos e implantados durante as décadas de 1980 e 1990 nas bibliotecas contribuíram não só para a o aprimoramento do processo de catalogação no âmbito automatizado, mas também para o desenvolvimento das redes bibliográficas. Assim, observa-se "[...] o esforço da biblioteconomia em direção ao aperfeiçoamento do acesso à produção bibliográfica mundial e representação pelo desenvolvimento das redes de informação, resultantes da aplicação da informática aos processos de organização

v.15

n.3

p.571-590

set./dez. 2017


bibliográfica" (CAMPELLO, 2006, p. 2).

Nesse contexto, a Ciência da Informação é responsável por investigar as propriedades e o comportamento da informação, o que permeia a origem, coleta, organização, armazenamento, recuperação, interpretação, transmissão, transformação e uso da informação (BORKO, 1968) e, por meio da catalogação, realiza a mediação entre a necessidade informacional do usuário e os documentos.

Dentre as descrições elaboradas pelo processo de catalogação, tem-se como foco deste trabalho o controle de autoridade, que se tornou um tema fundamental durante a década de 1980, quando bibliotecas passaram a automatizar seus catálogos com o intuito de realizar o compartilhamento e o intercâmbio de dados. O controle de autoridade é realizado a partir do trabalho de autoridade, que compreende: 1) a compilação, o registro e a manutenção dos dados de autoridade; 2) a verificação destes dados; e, 3) o uso de formas estabelecidas e autorizadas como pontos de acesso no catálogo da biblioteca (JIMENES PELAYO; GARCIA BLANCO, 2002). Esse conjunto de ações promove consistência e estabelece relações entre nomes, obras e temas (TAYLOR, 2006).

O trabalho de autoridade pode ser realizado de maneira individual e cooperativa. De acordo com Burke e Shorten (2010), bibliotecas de pequeno porte tendem a realizar o trabalho em nível local, enquanto bibliotecas de grande porte são mais propensas a fazer parte de redes de cooperação e de consórcios internacionais..

Neste cenário, os padrões de metadados são considerados como ferramentas essenciais para a representação e o acesso aos recursos informacionais (ALVES; SANTOS, 2013) e possibilitam o intercâmbio e o compartilhamento de dados em redes de cooperação e de consórcios internacionais, sobretudo em um contexto social e tecnológico em que a popularização das tecnologias da informação e da comunicação tem promovido o aumento expressivo de conteúdos disponibilizados e, gera a necessidade de integração de registros bibliográficos e de autoridade no ambiente da Web.

Os padrões de metadados têm influenciado uma nova perspectiva de infraestrutura para o intercâmbio de registros bibliográficos e para o controle bibliográfico universal, assim como têm criado condições para que metadados bibliográficos possam ser utilizados por outras comunidades da Web por meio do projeto Linked Open Data (LOD).

Esse novo ambiente tecnológico de aplicação repercutiu uma maior complexidade sobre os princípios de intercâmbio de registros bibliográficos e de autoridade, pois "Um padrão de metadados pode apresentar um bom esquema de descrição, mas pode proporcionar uma representação insuficiente se não forem utilizados esquemas de codificação para a unificação dos dados e valores [...]” (ALVES; SANTOS, 2013, p. 126). 
Por meio da descrição de valores de dados realizada a partir de elementos de metadados são estabelecidos vocabulários de valor que fornecem precisão, consistência, coerência e completude aos registros, e uma maior qualidade se comparados à descrição elaborada por usuários leigos (WILLER; DUNSIREE, 2013).

Neste contexto, foram desenvolvidos pela comunidade internacional de catalogadores projetos em busca do desenvolvimento de um serviço que cobre a agregação de registros de autoridade e harmonização de códigos de catalogação e padrões de metadados, com a premissa de que "O controle de acesso do futuro proporcionará diferenças sobre a adoção de distintos códigos de catalogação, formas de transliteração e diferenças culturais" (TILLET, 2000, p. 168, tradução nossa).

A comunidade internacional tem atuado na harmonização de padrões de representação em ambientes web por meio da conversão de registros bibliográficos e de autoridade em linguagens de estrutura e descrição, tais como o eXtensible Markup Language (XML) e o Resource Description Framewordk (RDF). Dentre as principais iniciativas, a International Federation of Library Associations and Institutions (IFLA), tem atuado na publicação de vocabulários de valor para os padrões, códigos, e diretrizes internacionais de catalogação como as diretrizes Resource Description and Access (RDA) e International Standard Bibliographic Description (ISBDs), e do modelo conceitual Functional Requirements for Bibliographic Records (FRBR) e suas extensões, que compreende os FRBRer, FRBRoo, FRAD e FRSAD. Esses vocabulários estão disponíveis por meio do sistema de gerenciamento Open Metadata Registry. ${ }^{1}$

Para a conversão dos dados são adotadas as recomendações de melhores práticas Linked Data e tecnologias da Web Semântica. Essas recomendações consistem em melhores práticas definidas pela comunidade internacional World Wide Web Consortium (W3C) para a publicação de conjuntos de dados que compreendem quatro princípios: 1) Use URIs como nomes para os recursos; 2) Use URIs HTTP como localização desses recursos; 3) Para melhorar a recuperação da informação, adote padrões na descrição e recuperação desses recursos, como RDF e SPARQL; e, 4) Estabeleça links entre os URIs para que outros recursos sejam recuperáveis (BERNERS LEE, 2006).

Os arquivos de autoridade disponibilizados no VIAF são estabelecidos por meio da unificação de valores adotados na descrição por cada Instituição cooperante, a fim de construir conjunto de valores que identificam e preservam variações linguísticas e nacionais. Por meio da formação de clusters de dados são estabelecidos vocabulários de valor na representação de elementos de autoridade a nível internacional.

Na catalogação, são adotados princípios, códigos, diretrizes e padrões que, por sua vez,

\footnotetext{
${ }^{1}$ Fonte: http://metadataregistry.org/
} 
são desenvolvidos e aprimorados de acordo com acordos e fundamentos internacionais. Para Silveira (2007, p. 11), "[...] os padrões e os conceitos devem ser compartilhados e respeitados por todos os países, uma vez que o controle bibliográfico universal depende do consenso global sobre a catalogação".

Esse contexto fornece destaque aos serviços estabelecidos por meio do consórcio VIAF, ao proporcionar a cooperação, o intercâmbio e a vinculação de arquivos de autoridade a nível internacional. $\mathrm{O}$ artigo caracteriza-se, como um estudo qualitativo de cunho exploratório realizado por meio de levantamento bibliográfico e revisão de literatura, com objetivo de apresentar os conceitos e fundamentos relacionados ao desenvolvimento, manutenção e funcionamento do consórcio VIAF.

\section{O CONTROLE DE AUTORIDADE NA CATALOGAÇÃo}

Durante a década de 1980 a evolução tecnológica potencializou a automação dos catálogos. Neste período o controle de autoridade tornou-se fundamental para a catalogação. O mesmo é obtido por meio do tratamento descritivo da informação enquanto processo adotado na definição de pontos de acesso, por meio do qual é realizada a descrição de elementos formais do objeto físico, que tem como produto final o registro bibliográfico e de autoridade (CATARINO; SOUZA, 2012).

O controle de autoridade é realizado por meio do trabalho de autoridade, que consiste em estabelecer relações entre diferentes pontos de acesso controlados, identificados do quadro 1, a fim de realizar a unificação de pontos de acesso e a devida associação com seus respectivos termos variantes (PASCUAL, 1999).

Quadro 1. Tipologia dos pontos de acesso

\begin{tabular}{|c|c|}
\hline \multicolumn{2}{|c|}{ Pontos de acesso } \\
\hline Não controlados & Controlados \\
\hline $\begin{array}{ll}\text { - } & \text { Títulos próprios } \\
\text { - } & \text { Palavras-chave }\end{array}$ & $\begin{array}{ll}\text { - } & \text { Nomes de pessoas } \\
\text { - } & \text { Nomes de entidades } \\
\text { - } & \text { Nomes geográficos } \\
\text { - } & \text { Títulos uniformes } \\
\text { - } & \text { Série } \\
\text { - } & \text { Materiais }\end{array}$ \\
\hline
\end{tabular}

Fonte: Adaptado de Sandín Enriquez (2011).

Com a automação das funções de catálogo foram estabelecidas redes de cooperação para o intercâmbio de registros bibliográficos, assim como contextualiza Santos e Pereira (2014, p. 126) 
A cooperação e o compartilhamento de informações catalográficas e bibliográficas entre diversas instituições no ambiente automatizado tornaram-se possível com o desenvolvimento e a adoção do MARC, o formato de intercâmbio de dados desenvolvido e testado pela LC, na década de 1960.

Nesse contexto, destacam-se os serviços estabelecidos por Bibliotecas e Agências Nacionais, diante do projeto Controle Bibliográfico Universal (CBU) idealizado pela IFLA e adotado pela UNESCO na década de 1970, que prevê a formação de uma rede internacional de controle e intercâmbio de informações bibliográficas (MACHADO, 2003). Entretanto, sua concretização requer o estabelecimento de um Controle Bibliográfico Nacional, por meio do qual as Bibliotecas e Agências Nacionais realizam a compilação de bibliografias nacionais e de medidas para o depósito legal, assim como, requerem o uso conjunto de padrões, códigos e diretrizes internacionais que permitam o intercâmbio de registros.

A interoperabilidade pode ser definida como "A capacidade de um sistema de hardware ou de software de se comunicar e trabalhar efetivamente no intercâmbio de dados com um outro sistema, geralmente de tipo diferente, projetado e produzido por um fornecedor diferente" (SAYÃO; MARCONDES, 2008, p. 136). Relacionada à interoperabilidade e às questões citadas anteriormente estão as múltiplas faces da interoperabilidade que necessitam ser atendidas. De acordo com Sayão e Marcondes (2008), as faces da interoperabilidade são a: 1) Interoperabilidade técnica - responsável por manter os sistemas de informação interoperáveis; 2) Interoperabilidade semântica - compreende a adoção de ferramentas semelhantes ou que apresentem correspondência na representação da informação; 3) Interoperabilidade política/humana - relaciona-se à decisão de promover ou não o acesso aos recursos informacionais; 4) Interoperabilidade intercomunitária - vincula-se às necessidades atreladas a pesquisa, acesso a informações quando elas são provenientes de diferentes fontes; 5) Interoperabilidade legal - remete aos aspectos legais e suas implicações na disseminação de recursos informacionais; 6) Interoperabilidade internacional - envolve a complexidade de comunicação em uma escala internacional, diante de aspectos linguísticos e culturais. Os metadados e padrões de metadados estão presentes nas faces sintáticas e semântica de interoperabilidade.

\section{INFRAESTRUTURA PARA CONSTRUÇÃo de VOCABULÁRIOS DE VALOR}

Segundo Breitman (2006) não existe uma definição universal para o conceito de metadados, o que para Alves e Santos (2013) representa um problema de pluralidade discursiva de significado. Isso porque o termo é adotado por diferentes áreas que trabalham com o tratamento e processamento de informação.

Na área de Ciência da Informação, Alves (2010, p. 47) define metadados como “dados que descrevem outros dados em um sistema de informação, com o intuito de identificar de

\begin{tabular}{l|l|l} 
v.15 & n.3 \\
\hline
\end{tabular}

p.571-590
set./dez. 2017 
forma única uma entidade (recurso informacional) para sua posterior recuperação”. Já os padrões de metadados especificam os elementos para a descrição de recursos, e são definidos como "[...] estruturas de descrição constituídas por um conjunto predeterminado de metadados (atributos codificados ou identificadores de uma entidade) metodologicamente construídos e padronizados" (ALVES, 2010, p. 47).

Padrões de metadados são adotados na elaboração de registros bibliográficos para o propósito específico de intercâmbio de registros, o que requer o uso de formato de metadados ricos que, segundo Alves (2005, p. 140) "[...] proporcionam uma representação completa e detalhada de um recurso informacional".

Os padrões de metadados ricos são compostos por padrões de estrutura de metadados descritivos e padrões de conteúdo. Os padrões de estrutura de metadados descritivos definem conjuntos de elementos considerados importantes para descrever o recurso, sua repetibilidade e ordem, e os padrões de conteúdo estabelecem regras de entrada em um campo de metadados pela sintaxe (CARLOS; ZAFALON, 2013).

Willer e Dunsiree (2013) consideram que os esquemas de metadados fornecem consistência à estrutura, pois determinam quais aspectos de um recurso devem ser descritos e como os dados devem ser armazenados, enquanto as regras de catalogação oferecem consistência e coerência ao conteúdo dos metadados..

Registros bibliográficos e de autoridade podem ser interoperáveis, desde que ancorados em tecnologias apropriadas. Desta forma, a International Federation of Library Associations and Institutions (IFLA) tem atuado para que padrões de metadados adotados por bibliotecas e instituições do patrimônio cultural tenham infraestrutura compatível com as tecnologias da Web Semântica, na qual, o estudo apresentado por Willer e Dunsiree (2013) discorre sobre as dificuldades encontradas em estabelecer metadados bibliográficos em formato RDF. A principal dificuldade apresentada por Willer e Dunsiree (2013) envolve aspectos tecnológicos e representacionais apresentados por Alves e Santos (2013, p. 120), de forma que seja estabelecida integração entre:

\footnotetext{
Modelagem conceitual do sistema, determinação dos requisitos funcionais, mapeamento dos elementos essenciais, determinação da estrutura de descrição do esquema, determinação dos espaços de valor e determinação dos esquemas de codificação para padronização dos espaços de valor.
}

O modelo conceitual Functional Requirements for Bibliographic Records (FRBR), traduzido para a língua portuguesa como Requisitos Funcionais para Registros Bibliográficos, também desenvolvido pela IFLA entre os anos de 1992 e 1998, define a partir do modelo conceitual Entidade-Relacionamento entidades, atributos e os possíveis relacionamentos entre registros bibliográficos e de autoridade (TILLET, 2007). Entidades são identificadas em 3 grupos: 
Grupo 1: Obra, expressão, manifestação e item;

Grupo 2: Pessoas e organizações;

Grupo 3: Conceito, objeto, evento, lugar e entidades do grupo 1.

O novo código de catalogação Resource Description and Access (RDA), que deve substituir o Anglo-American Cataloguing Rules (AACR2), fornece instruções de como registrar dados a partir do modelo conceitual Functional Requirements for Authority Data (FRAD) (SALGADO; SILVA, 2013), projetado para

[...] criar um quadro de referência claramente definido, estruturado, de modo a relacionar os dados dos registros de autoridade às necessidades dos usuários de tais dados; assim como auxiliar na avaliação do potencial de compartilhamento internacional de dados de autoridade, não restrito ao universo bibliográfico (MEY; SILVEIRA 2009, p. 38).

Uma das questões fundamentais para o controle bibliográfico nacional e internacional é o controle de autoridade que estabelece, a partir de pontos de acesso controlados, a identificação de nomes de pessoas, nomes de entidades, nomes geográficos, títulos uniformes, série e materiais, pois, de acordo com a Declaração de Princípios (INTERNATIONAL FEDERATION OF LIBRARY ASSOCIATIONS AND INSTITUTIONS, 2009) registros de autoridade são adotados a fim de permitir o controle de formas autorizadas e variações na identificação de pontos de acesso. Deste modo, compreende-se que formas autorizadas, formas variantes e relacionadas de nome devem coexistir por meio de relacionamentos que estabeleçam uniformidade ao acesso, e a representação de pontos de acesso deve considerar variações linguísticas e culturais (INTERNATIONAL FEDERATION OF LIBRARY ASSOCIATIONS AND INSTITUTIONS, 2004).

Assim como o controle bibliográfico, o controle de autoridade é estabelecido por meio do uso comum de ferramentas especificas que proporcionem ao sistema de informação estrutura, conteúdo, modelos e princípios assim como ilustrado na figura 1. 


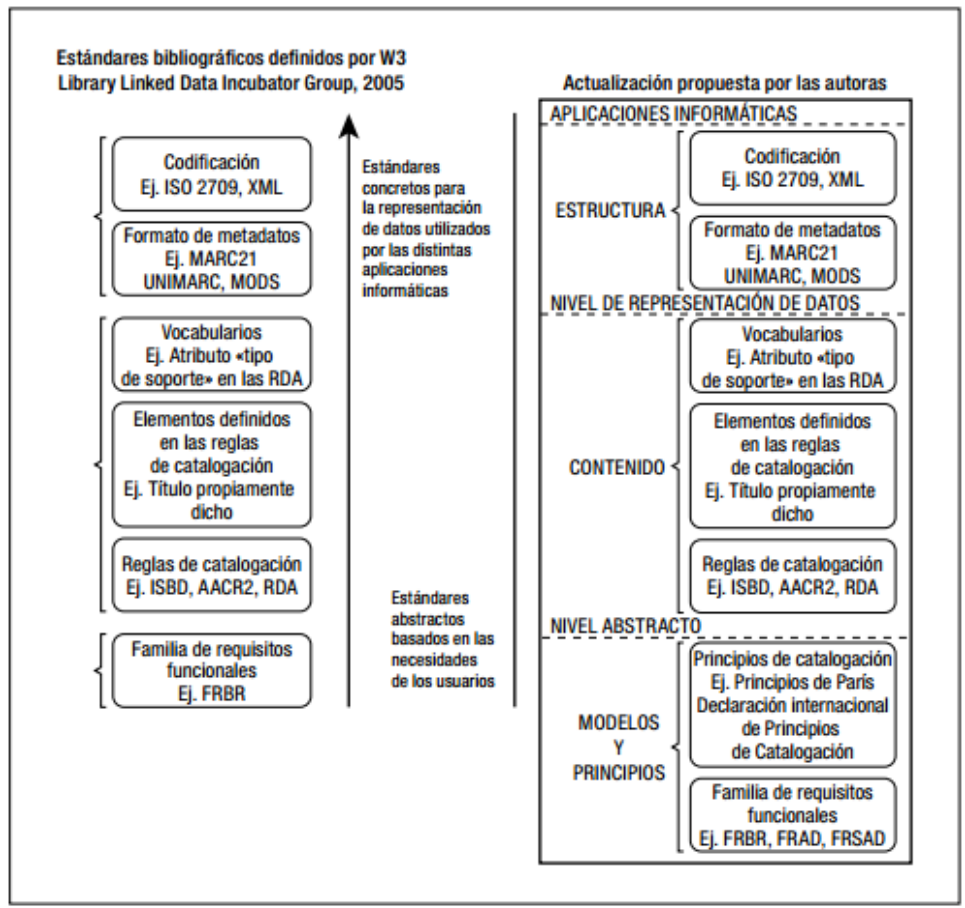

Figura 1. Padrões para implementação do controle bibliográfico Fonte: Picco e Ortiz Repiso (2012, p. 149).

A infraestrutura apresentada na figura possibilita que $o$ intercâmbio e $o$ compartilhamento dos dados sejam realizados de forma consistente e sejam passiveis de uso e reuso na representação e recuperação da informação.

\section{COOPERAÇÃO INTERNACIONAL E UNIFICAÇÃO DE VALORES POR CLUSTER NO VIAF}

No VIAF, a cooperação internacional é estabelecida a partir da formação de registros por cluster, que identificam variações nacionais, linguísticas e culturais na forma de descrição de valores, a partir da agregação de elementos de autoridade que compõem padrões de metadados.

Atualmente, o VIAF conta com 46 bibliotecas e agências nacionais cooperantes localizadas em diferentes países (VIRTUAL INTERNATIONAL AUTHORITY FILE, 2017) apresentados na figura 2 . 


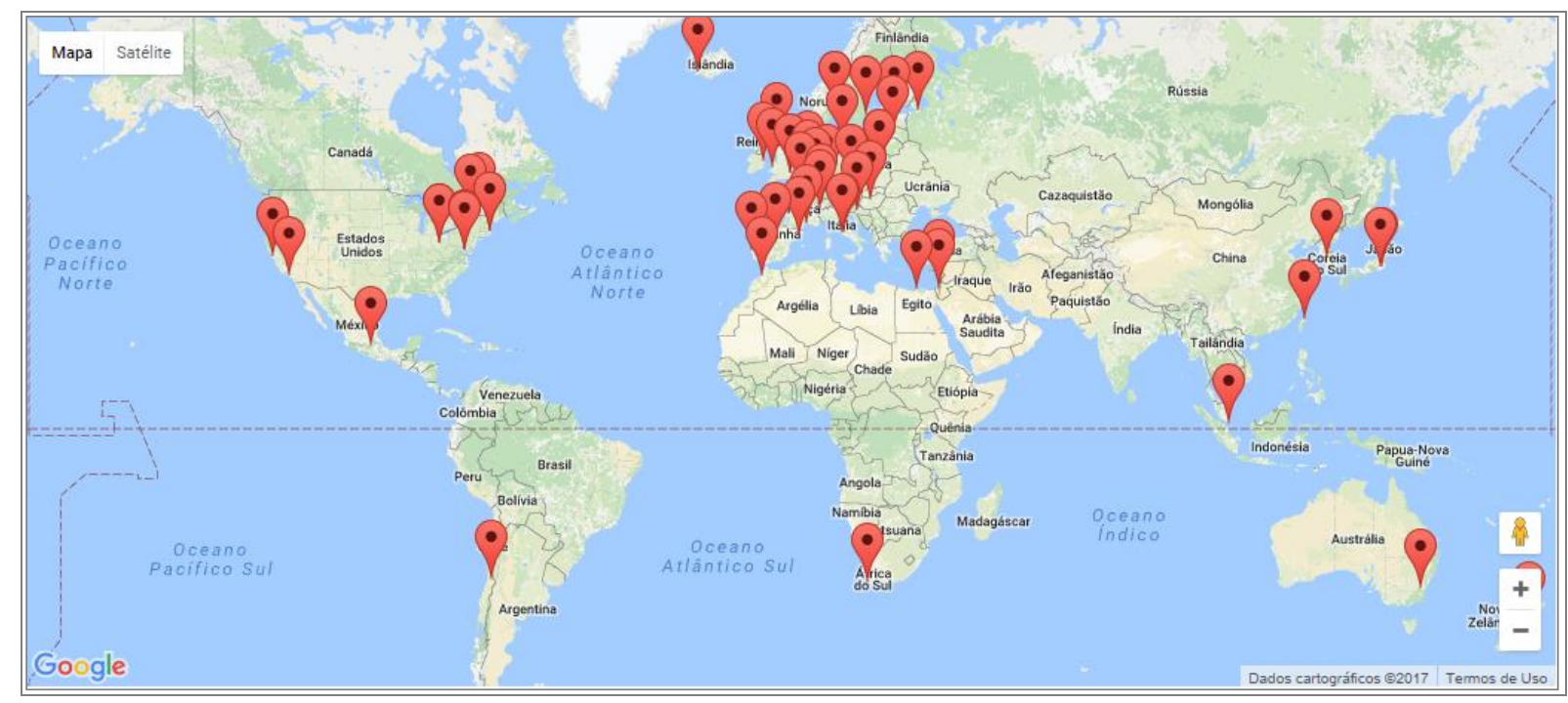

Figura 2. Mapa de países que participam do consórcio VIAF Fonte: Virtual International Authority File (2017).

Os registros que integram o consórcio VIAF, por serem metadados elaborados no âmbito de Bibliotecas e Agências Nacionais, são estruturados a partir do formato de intercâmbio MARC para dados de autoridade. Assim como é apresentado por Isaac et al. (2011), registros de bibliotecas consistem em declarações sobre as obras, nas quais cada declaração é composta por um elemento da entidade e seu respectivo valor, o que abrange padrões de estrutura, metadados descritivos e padrões de conteúdo, assim como requer o uso de vocabulários de valor.

Vocabulários de valor são adotados na elaboração de metadados por proporcionar maior consistência na descrição do conteúdo de sistemas de recuperação da informação (NATIONAL INFORMATION STANDARDS ORGANIZATION, 2005). Esses vocabulários podem ser definidos como uma lista controlada de valores permitidos para um elemento, o que proporciona controle terminológico na descrição (ISAAC et al., 2011).

A interface de busca do sistema permite que as buscas sejam realizadas por campos denominados:

- Todos os campos;

- Todos os títulos;

- Nomes corporativos;

- Nomes geográficos;

- Nomes pessoais;

- Obras;

- Expressões;

- Títulos preferenciais;

- Títulos bibliográficos. 
No VIAF, os registros são agrupados automaticamente por meio de um algoritmo de comparação entre os registros nacionais e a base WorldCat, com base nos FRBR (FARINOS, 2014). Esse agrupamento estabelece a formação de registros por cluster dos elementos de autoridade, que contém a descrição das entidades e as variações, com a devida identificação do país de origem da instituição onde os metadados foram elaborados. A cada registro, os metadados são apresentados pelos campos apresentados no quadro 2 .

Quadro 2. Campos de apresentação de metadados adotados no VIAF

\begin{tabular}{|l|l|}
\hline \multicolumn{1}{|c|}{ Campo } & \multicolumn{1}{c|}{ Descrição } \\
\hline Forma preferida & Apresenta a entrada autorizada adotada por cada unidade cooperante. \\
\hline Forma de nome alternativo & $\begin{array}{l}\text { Identifica variações e formas alternativas de descrição adotadas por } \\
\text { cada unidade cooperante. }\end{array}$ \\
\hline Nome relacionado & $\begin{array}{l}\text { Apresenta nomes relacionados ao registro de autoridade no registro } \\
\text { bibliográfico. } \\
\text { Exemplo: coautores; instituições de filiação; entre outros. }\end{array}$ \\
\hline Obra & $\begin{array}{l}\text { Identifica os tipos do recurso representado no registro bibliográfico } \\
\text { relacionado ao registro de autoridade. }\end{array}$ \\
\hline Coautores selecionados & Apresenta nomes de coautores. \\
\hline País de publicação & $\begin{array}{l}\text { Apresenta a identificação do local de publicação informado no } \\
\text { registro bibliográfico. }\end{array}$ \\
\hline Estatísticas de Publicações & Apresenta a representação gráfica de estatísticas de publicações. \\
\hline Editores selecionados & Apresenta informações sobre o editor. \\
\hline Sobre & $\begin{array}{l}\text { Descreve informações pessoais por nacionalidade e idioma, assim } \\
\text { como apresenta links externos. }\end{array}$ \\
\hline Registros & $\begin{array}{l}\text { Disponibiliza acesso aos registros em formato MARC record; VIAF } \\
\text { Cluster XML; RDFS; e, JSON. }\end{array}$ \\
\hline Histórico do ID no VIAF & $\begin{array}{l}\text { Apresenta registros adicionados e excluídos por cada unidade } \\
\text { cooperante, com informação de data e a hora. }\end{array}$ \\
\hline
\end{tabular}

Fonte: Adaptado de Virtual International Authority File (2017).

A cada cluster gerado pelo sistema, um Uniform Resource Identifiers (URI) e um link permanente são atribuídos, oferecendo maior consistência e integridade aos registros apresentados na figura 3. Segundo Marcondes (2012), as URIs identificam recursos informacionais disponíveis em formato digital e recursos reais, quando estes não estão em formato digital e são substituídos por sua representação. 


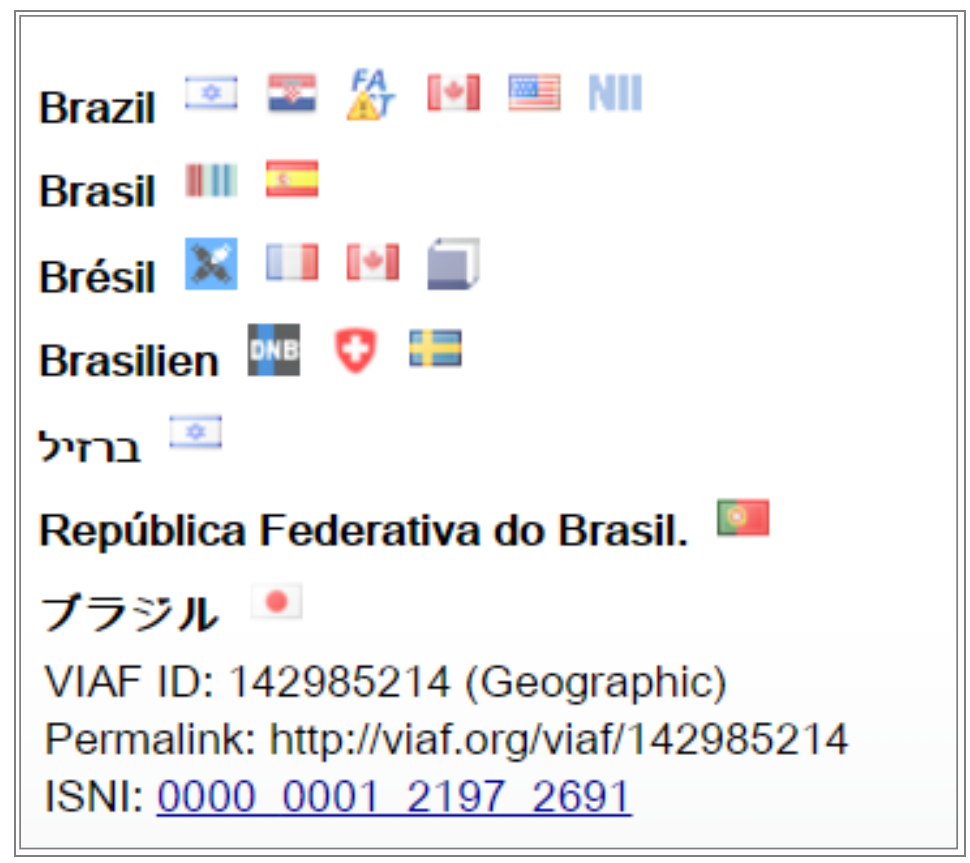

Figura 3. Cluster VIAF para Nome Geográfico

Fonte: Virtual International Authority File (2017).

A interface de consulta aos registros disponibiliza arquivos em formato MARC-21 record, VIAF Cluster in XML, RDF record e Just Links em JSON, que estabelecem as relações sintáticas e semânticas, no quais o registro em estrutura RDF dos arquivos disponibilizados no VIAF permite que os elementos de metadados do MARC autoridade sejam mapeados e interoperáveis com outros padrões. A correspondência entre elementos definidos por diferentes padrões de metadados pode ser viabilizada por conversão, assim como é apresentado por Library of Congress (2008) e Chan e Zeng (2006) que descrevem métodos de estabelecer correspondência entre elementos do formato MARC $21 \mathrm{com}$ os do Dublin Core.

Na agregação dos elementos, a visualização de semelhanças e diferenças na forma descrição na entrada autorizada adotada por cada unidade cooperante é identificada pela bandeira do país, conforme apresentado na figura 4. 


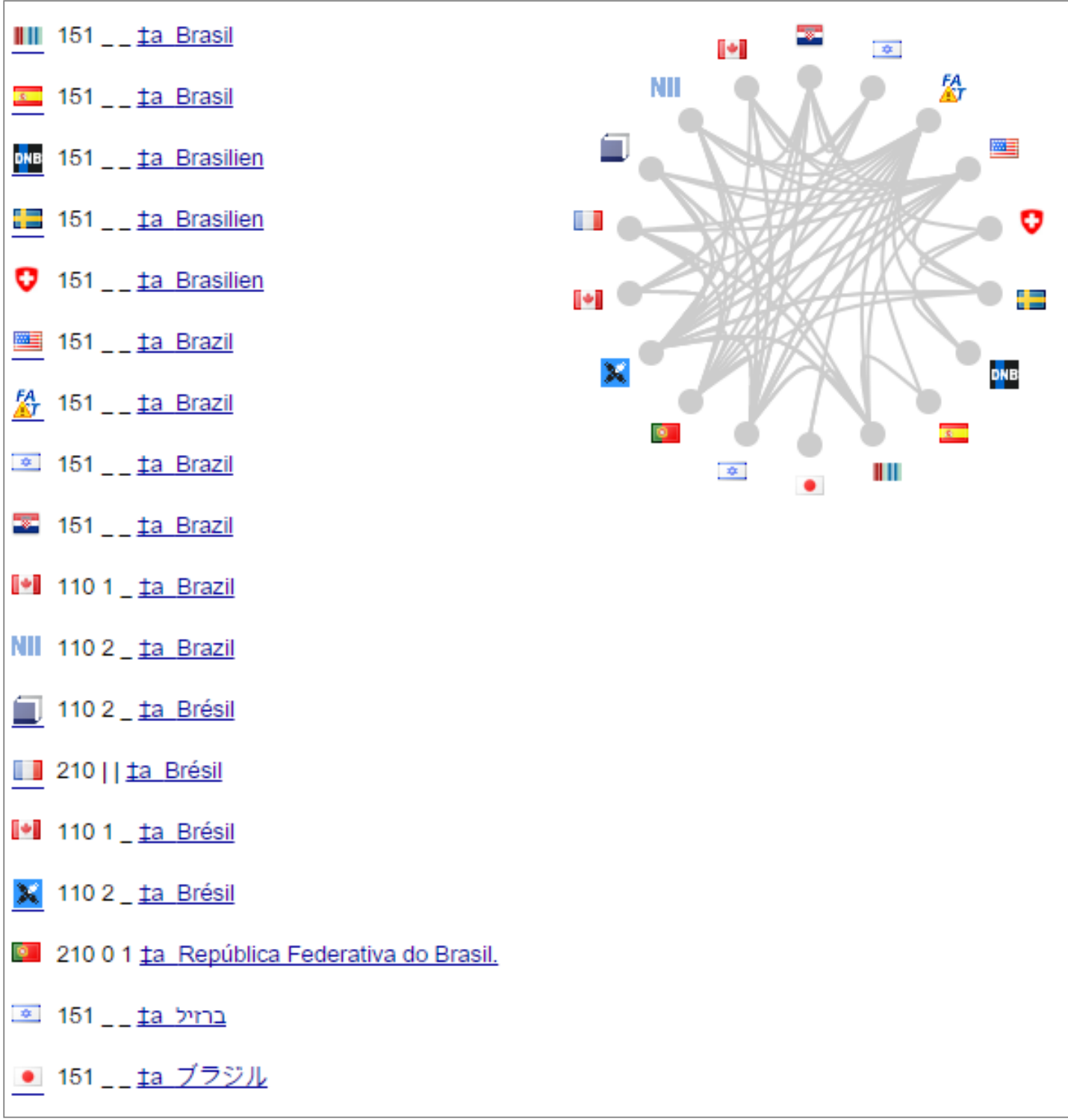

Figura 4. Cluster VIAF Nome Geográfico

Fonte: Virtual International Authority File (2017).

A representação gráfica apresentada na figura 4 é estabelecida por meio de uma teia de dados, que além de proporcionar uma melhor visualização das relações semânticas, permite identificar a entrada ou forma de descrição do valor adotada em cada país.

Os conjuntos de dados do VIAF estão integrados ao projeto Linked Open Data. Neste projeto a comunidade internacional tem atuado na publicação de dados vinculados de acesso aberto. Na figura 5 é possível identificar que os dados do VIAF estão próximos aos dados da DBpedia ${ }^{2}$, um conjunto de dados estruturados da Wikipédia considerado o principal conjunto de dados na nuvem.

\footnotetext{
${ }^{2}$ Fonte: $<$ http://wiki.dbpedia.org/>.
} 


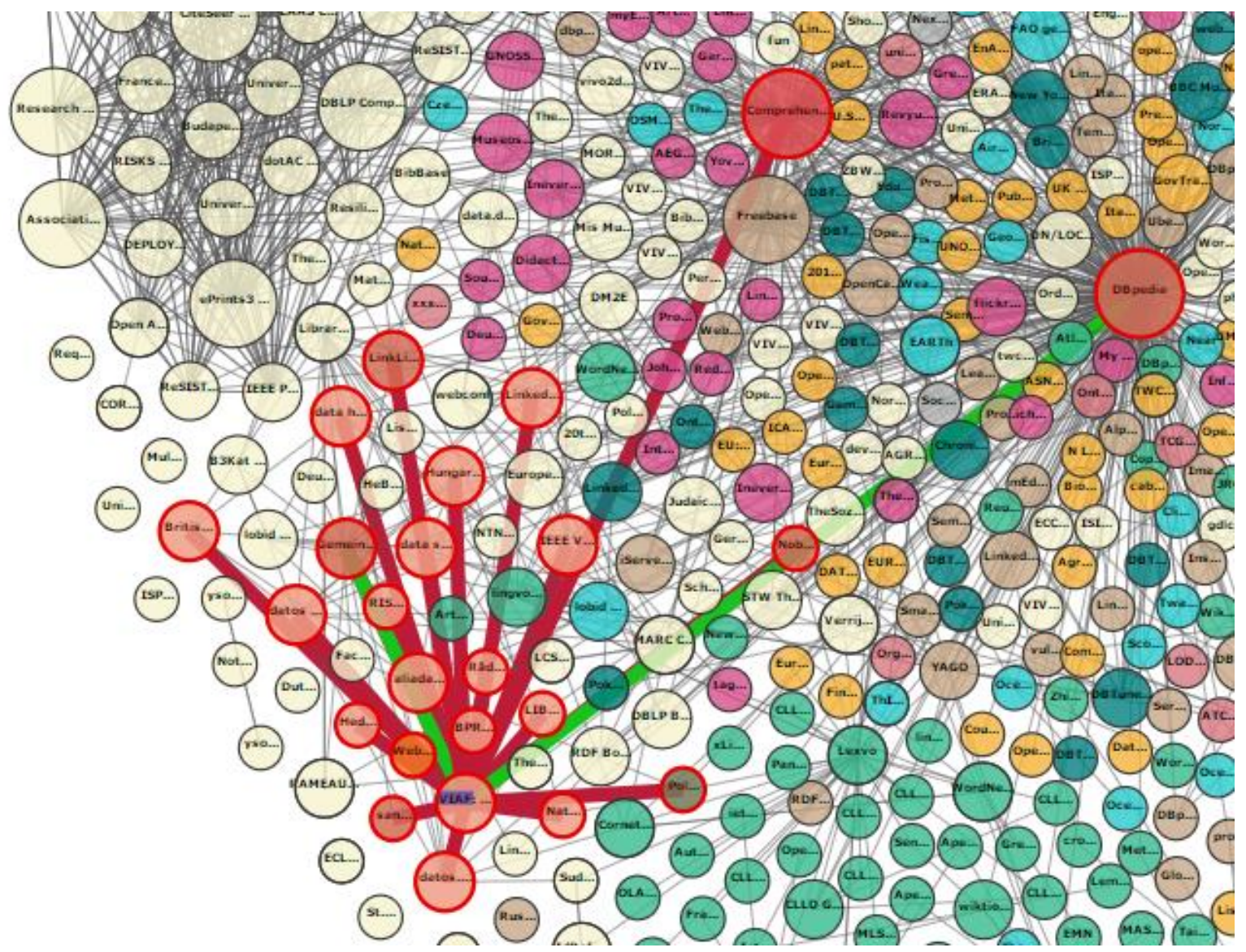

FIGURA 5. Diagrama Linked Open Data

Fonte: Adaptado de Linked Open Data (2017, não paginado).

Os conjuntos de dados são disponibilizados na nuvem a partir de sua infraestrutura e da vinculação com outros conjuntos de dados, porém a representação gráfica dos conjuntos de dados no diagrama de LOD requer critérios de qualidade, o que indica a qualidade e consistência dos arquivos de autoridade na teia de dados.

\section{CONSIDERAÇÕES FINAIS}

As tecnologias da informação e da comunicação (TIC) têm provocado profundas transformações nas as técnicas de tratamento descritivo da informação. Para a Catalogação descritiva, a evolução tecnológica potencializou o aprimoramento das ferramentas de trabalho, o que deu origem a uma nova perspectiva de infraestrutura tecnológica para o intercâmbio de metadados, que tem sido aplicada nos novos padrões de metadados em desenvolvimento, assim como no mapeamento de padrões já adotados no domínio bibliográfico, criando condições para a concretização da catalogação única idealizada por Charles Jewett e do Controle Bibliográfico Universal pela IFLA. set./dez. 2017 
No domínio bibliográfico, assim como afirmam Willer e Dunsiree (2013), desde a criação das 91 regras de Panizzi para o catálogo do Museu Britânico, as bibliotecas têm acumulado há mais de 150 anos uma vasta quantidade de registros bibliográficos elaborados a partir de técnicas de tratamento descritivo da informação, que até pouco tempo eram restritos ao ambiente das Bibliotecas e às funções de catálogo. Desta forma, a atribuição de valor adotada na definição de pontos de acesso, mesmo quando ancorados em normas internacionais, podem apresentar incompatibilidades na descrição de valores.

O controle de autoridade consiste em uma importante ferramenta de padronização e controle terminológico de grande contribuição para qualidade do funcionamento de sistemas de busca e recuperação da informação, nos quais a unificação de valores estabelecida a partir do consórcio Virtual International Authority File (VIAF) contribui na agregação de valores cobrindo variações linguísticas e culturais, assim como na soma de metadados elaborados por instituições que atuam no controle bibliográfico nacional, o que proporciona uma maior cobertura sobre as publicações em cada país.

Deste modo, o VIAF pode ser considerado como uma fonte confiável de registos de autoridade como vocabulário de valor para todo o domínio bibliográfico, uma vez que integra registros elaborados por instituições reconhecidas em cada país, e que abrange variações linguísticas em nível internacional, seguindo os princípios da catalogação única, assim como estende sua perspectiva de uso dos registros para outras comunidades da Web de dados.

\section{REFERÊNCIAS}

ALVES, R. A. V. Metadados como elementos do processo de catalogação. 2010. 132 f. Tese (Doutorado em Ciência da Informação) - Universidade Estadual Paulista, Marília, 2010. Disponível em: < http://www.marilia.unesp.br/Home/PosGraduacao/CienciadaInformacao/Dissertacoes/alves_rachel.pdf >. Acesso em: 30 jul. 2015.

ALVES, R. A. V. Web Semântica: uma análise focada no uso de metadados. 2005. Dissertação (Mestrado em Ciência da Informação) - Faculdade de Filosofia e Ciências, Universidade Estadual Paulista, Marília, 2005. Disponível em: < http://www.marilia.unesp.br/Home/PosGraduacao/CienciadaInformacao/Dissertacoes/alves_rcv_me_mar.pdf >. Acesso em: 23 abr. 2015.

ALVES, R. C. V; SANTOS, P. L. V. A. C. Metadados no domínio bibliográfico. Rio de Janeiro: Intertexto, 2013.

ANGJELI, A.; MAC EWAN, A.; BOULET, V. ISNI and VIAF: Transforming ways of trustfully consolidating identities. In: IFLA GENERAL CONFERENCE AND ASSEMBLY, 80, 2014. Proceedings... Lyon: IFLA, 2014.

BERNEERS, LEE, T. Linked data. 2006. Disponível em: < https://www.w3.org/DesignIssues/LinkedData.html >. Acesso em: 20 out. 2016.

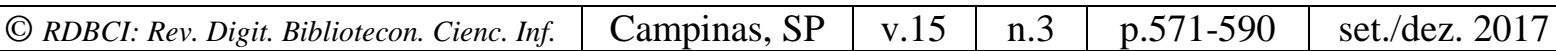


BORKO, H. Information Science: What is it? American documentation, p. 3-5, 1968

BREITMAN, K. Web Semântica: A internet do futuro. Rio de Janeiro: LTC, 2006. 190 p.

BURKE, S.K.; SHORTEN, J. Name authority work today: A comparison of types of academic libraries. Library resources and technical servives, n.1, v.54, 2010.

CAMPELLO, B. Introdução ao controle bibliográfico. Brasília: Briquet de Lemos, 2006.

CARLOS, K. V.; ZAFALON, Z. R. Metadados e Interoperabilidade em Bibliotecas Nacionais Sul-Americanas. In: ENCONTRO INTERNACIONAL DE CATALOGADORES, 9.; ENCONTRO NACIONAL DE CATALOGADORES, 2., 2013, Rio de Janeiro. Anais... Rio de Janeiro: Fundação Biblioteca Nacional do Brasil, 2013. Disponível em: < http://www.abinia.org/catalogadores/62-210-1-PB.pdf >. Acesso em: 01 ago. 2015.

CATARINO, M. E.; SOUZA, T. B. A representação descritiva no contexto da Web Semântica. Transinformação, v.24, n.2, p.77-90, 2012. Disponível em: < http://www.scielo.br/scielo.php?pid=S0103-7862012000200001\&script=sci_arttext $>$. Acesso em: 15 jun. 2015.

CHAN, L. M.; ZENG, M. L. Metadata Interoperability and Standardization - a study of methodology part I: achieving interoperability at the schema level. D-Lib Magazine, v.12, n. 6, 2006. ISSN 1082-9873. Disponível em: < http://www.dlib.org/dlib/june06/chan/06chan.html >. Acesso em: 15 jun. 2015.

GARZON-FARINOS, F. G. El registros de autoridades personales tras la aparición del Web. Tese (Doutorado) - Universitat Politècnica de València, Valencia, 2014. Disponível em: <http://eprints.rclis.org/24571/>. Acesso em: 15 ago. 2015.

INTERNATIONAL FEDERATION OF LIBRARY ASSOCIATIONS AND INSTITUTIONS. Declaração dos princípios internacionais de catalogação. 2009. Disponível em: < http://www.ifla.org/files/assets/cataloguing/icp/icp_2009-pt.pdf >. Acesso em: 15 jul. 2015.

INTERNATIONAL FEDERATION OF LIBRARY ASSOCIATIONS AND INSTITUTIONS. Directrices para Registros de Autoridad y Referencias. 2004. ISBN 8436938380. Disponível em: < http://archive.ifla.org/VII/s13/garr/garr-es.pdf >. Acesso em: 20 ago. 2015.

ISAAC, A. et al. Library Linked Data Incubator Group: Datasets, Value Vocabularies, and Metadata Element Sets. W3C Incubator Group Report, 2011. Disponível em: < http://www.w3.org/2005/Incubator/lld/XGR-lld-vocabdataset-20111025/ >. Acesso em: 1 ago. 2015.

JIMENEZ PELAYO, J.; GARCÍA BLANCO, R. EI Catálogo de autoridades: Creación y gestión en unidades documentales. Gijón: TREA, 2002. ISBN 8497040279. Disponível em: < http://biblioteca.udgvirtual.udg.mx/eureka/pudgvirtual/jimenezP.pdf $>$. Acesso e 21 jan. 2016 
LIBRARY OF CONGRESS. Dublin Core to MARC Crosswalk: Network Development and MARC Standards Office Library of Congress. 2008. Disponível em: < http://www.loc.gov/marc/marc2dc.html >. Acesso em: 11 jul. 2015.

MACHADO, A. M. N. Informação e controle bibliográfico: um olhar sobre a cibernética. São Paulo: Ed. UNESP, 2003.

MARCONDES, C. H. "Linked data" - dados interligados - e interoperabilidade entre arquivos, bibliotecas e museus na web. Encontros Bibli: revista eletrônica de biblioteconomia e ciência da informação, v.17, n.34, p.171-192, 2012. ISSN 1518-2924. DOI: $10.5007 / 1518-2924.2012 v 17 n 34 p 171$. Disponível em: < https://periodicos.ufsc.br/index.php/eb/article/view/1518-2924.2012v17n34p171/22782 >. Acesso em: 14 jul. 2015.

MEY, E. S. A.; SILVEIRA, N. C. Considerações teóricas aligeiradas sobre a catalogação e sua aplicação. InCID: Revista de Ciência da Informação e Documentação, v.1, n.1, p. 125137, 2010. Disponível em: <

http://www.brapci.inf.br/_repositorio/2010/12/pdf 4520d684ee_0014017.pdf $>$. Acesso em: 1 ago. 2015.

MEY, E. S. A.; SILVEIRA, N. C. Catalogação no plural. Brasília: Briquet de Lemos, 2009.

MILLER, M.; DUNSIRE, G. Publishing bibliographic element sets and value vocabularies.

In: Bibliographic Information Organization in the Semantic Web. [S.1.]:

Chandos Publishing, 2013. p.137-198.

NATIONAL INFORMATION STANDARDS ORGANIZATION. ANSI/NISO. Z39.19-

2005. Guidelines for the Construction, Format, and Management of Monolingual Controlled Vocabularies. Disponível em: < http://www.niso.org/standards/z39-19-2005/ >. Acesso em: 01 ago. 2015.

ONLINE COMPUTER LIBRARY CENTER. VIAF: Virtual International Authority File. 2015. Disponível em: < http://www.oclc.org/viaf.en.html >. Acesso em: 13 maio 2015.

ONLINE Dictionary for Library and Information Science. 2016. Disponível em: < http://www.abc-clio.com/ODLIS/odlis_i.aspx >. Acesso em: 20 out. 2016

PASCUAL, C. H. El Control de Autoridades. Anales de Documentación, n. 2, 1999, p.121136.

PASTOR SANCHEZ, J.A.; MARTÍNEZ MÉNDEZ, F.J.; RODRÍGUES MUNOZ, J.V. Aplicación de SKOS para la interoperabilidad de vocabularios controlado en el entorno de Linked Open Data. El profesional de la información, v.21, n.3, p.245-253, 2012.

PICCO, P.; ORTIZ REPISO, V. RDA, el nuevo código de catalogación: cambios y desafios para su aplicación. Revista española de documentação científica, n.35, v.1, p.145-173, 2012. ISSN 0210-0614. Disponível em: < 
http://www.ugr.es/ rruiz/docencia/automatizada/RDA_Nuevo_codigo_de_catalogacion.pdf >. Acesso em: 20 out. 2016.

SALGADO, D. M. S.; SILVA, J. F. M. AACR2 X RDA: breves reflexões acerca dos registros de autoridade. In: CONGRESSO BRASILEIRO DE BIBLIOTECONOMIA, DOCUMENTAÇÃO E CIÊNCIA DA INFORMAÇÃO, 25., 2013, Florianópolis. Anais... São Paulo: FEBAB, 2013.

\section{SANDÍN ENRIQUEZ, D. El Fichero Internacional Virtual de Autoridades (VIAF):}

Estado de la cuestión, evaluación y propuestas de mejora. 2011. 110f. Dissertação (Máster en Sistemas de Información Digital) - Facultad de Traducción y Documentación, Universidad de Salamanca, Salamanca, 2011.

SANTOS, P. L. V. A. C.; PEREIRA, A. M. Catalogação: breve história e contemporaneidade. Niterói: Intertexto, 2014.

SAYÃO, L. F. Padrões para bibliotecas digitais abertas e interoperáveis. Encontros Bibli:

Revista eletrônica de Biblioteconomia e Ciência da Informação, n. esp., 2007. Disponível em: < https://periodicos.ufsc.br/index.php/eb/article/view/1518-2924.2007v12nesp1p18/436 $>$. Acesso em: 20 jul. 2015.

SAYÃO, L. F.; MARCONDES, C. H. O desafio da interoperabilidade e as novas perspectivas para as bibliotecas digitais. TransInformação, v. 20, n. 2, p.133-148, maio/ago. 2008. Disponível em: < http://periodicos.puccampinas.edu.br/seer/index.php/transinfo/article/view/530/510 >. Acesso em: 15 jul. 2015.

SILVEIRA, N. C. Análise do impacto dos Requisitos Funcionais para Registros Bibliográficos (FRBR) nos pontos de acesso de responsabilidade pessoal. Dissertação (Mestrado em Ciência da Informação) - Pontifícia Universidade Católica de Campinas, 2007. Disponível em: < http://www.bibliotecadigital.puccampinas.edu.br/tde_busca/arquivo.php?codArquivo=343 >. Acesso em: 01 ago. 2015.

TAYLOR, A.G. Introduction to Cataloging and Classification. 10th Ed. Westport: Libraries Unlimited, 2006.

TILLET, B.B. Authority Control at the International Level. Association for Library Collection e Technical Services, v.44, n.3, 2000.

TILLET, B.B. Base Virtual Internacional de Autoridade. Pretoria: IME ICC, 2007. Disponível em: < http://www.referenceglobal.com/doi/abs/10.1515/9783598441028.2.201 > Acesso em: 05 abr. 2015.

VIRTUAL INTERNATIONAL AUTHORITY FILE. 2017. Disponível em:< https://viaf.org/ >. Acesso em: 04 maio de 2016. 

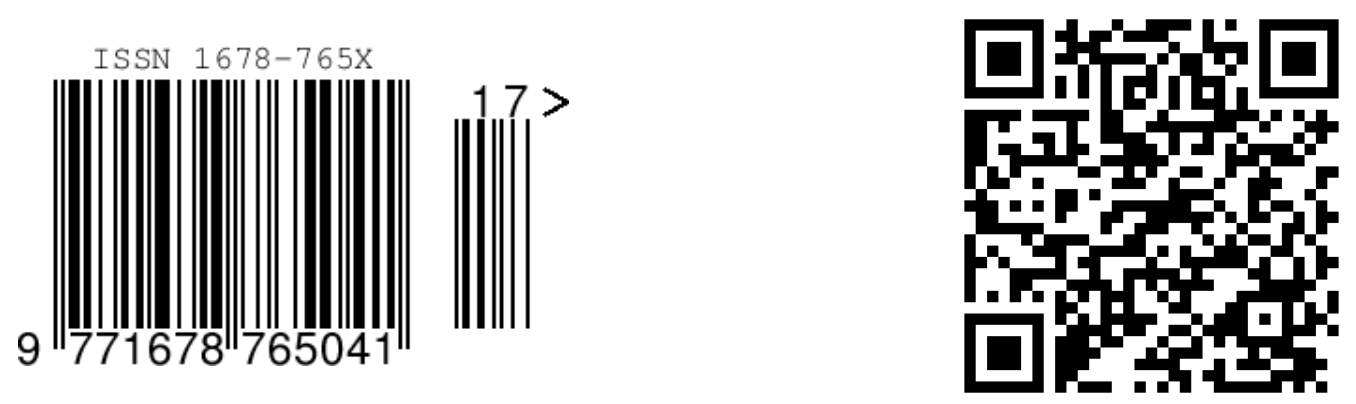